tivists have not dived down into the æther, as someone must ultimately dive, and dissected out the intrinsic speed of its turbulence-which is really the fundamental velocity in existence-they have utilised the more obvious and conspicuous consequence of this fundamental speed, viz. the uniform velocity with which the æther can convey a great variety of signals. Indeed their attachment to appliances like clocks and rods has led them to pay almost undue reverence to the æther's power of transmitting waves at a high and otherwise unattainable speed. It is true that these waves are among our methods of receiving and conveying information; but too much attention may be paid to the mere reception of information; and what is spoken of as "warping" is not limited to space alone. For some philosophers speak as if the duration of an event could be extended by merely delaying the reception of the news of its end; as if we could prolong a man's life by evading the tidings of his death, and might be entitled to say, without absurdity, that a man who died at seventy had lived seventy-one years and a lot of miles, if we had travelled so far that the messenger took a year to reach us. That such things can be gravely uttered is surely a tribute to the beauty and complexity of the mathematical scheme which can temporarily so warp the judgment even of the most competent.

If I am wrong in this I share a fraction of rashness with the admirable audacity of Einstein in the Weltmacht oder Niedergang sort of attitude which he takes up about his predicted shift of spectral lines. I feel a doubt whether those lines will be found shifted-at least when the observation is made outside a strong gravitational field-but I should be quite content either way, and would not think of asking anyone to abandon the method of relativity on that account. ${ }^{2}$

For undoubtedly general relativity, not as a philosophic theory but as a powerful and comprehensive method, is a remarkable achievement; and an ordinary physicist is full of admiration for the equations and the criteria, borrowed from hyperGeometers, applied by the genius of Einstein, and expounded in this country with unexampled thoroughness and clearness by Eddington. But, notwithstanding any temptation to idolatry, a physicist is bound in the long run to return to hts right mind; he must cease to be influenced unduly by superficial appearances, impracticable measurements, geometrical devices, and weirdly ingenious modes of expression; and must remember that his real aim and object is absolute truth, however difficult of attainment that may be, that his function is to discover rather than to create, and that beneath and above and around all Appearances there exists a universe of full-bodied, concrete, absolute, Reality.

2 Since the above paragraph was in type a Circular, dated January, 1921, has reached me from Prof. Slipher, of the Lowell Observatory a Flagstaff, Arizona, recording an extravagant rate of recession-something like a thousand miles a second-for two specified nebula, presumably of the spiral class. Rut both nebulx are reported to have large and billiant nuc ei ; and if the concentration of their aggregate mass were sufficientthat is, if their $M / R$ or $\rho^{k_{2}}$ were some two or three thousand's of times greater than that of our sun, and therefore of a totally different order from that of our stellar system-the observation could be interpreted, not as recessic $n$, but as an Einstein shift of speciral lines.

\title{
Electricity and Gravitation
}

By Prof. H. Weyl.

[Translated by Dr. Robert W. Lawson.]

MODERN physics renders it probable that the D only fundamental forces in Nature are those which have their origin in gravitation and in the electromagnetic field. After the effects proceeding from the electromagnetic field had been co-ordinated by Faraday and Maxwell into laws of striking simplicity and clearness, it became necessary to attempt to explain gravitation also on the basis of electromagnetism, or at least to fit it into its proper place in the scheme of electromagnetic laws, in order to arrive at a unification of ideas. This was actually done by $\mathrm{H}$. A. Lorentz, G. Mie, and others, although the success of their work was not wholly convincing. At the present time, however, in virtue of Einstein's general theory of relativity, we understand in principle the nature of gravitation, and the problem is reversed. It is necessary to regard electromagnetic phenomena, as well as gravitation, as an outcome of the geometry of the universe. I believe that this is possible when we liberate the world-geometry (on which Einstein based his theory) from an inherent inconsistency, which is still associated with it as a consequence of our previous Euclidean conceptions. NO. 2677 , VOL. IO6]
The great accomplishment of the theory of relativity was that it brought the obvious principle of the relativity of motion into harmony with the existence of inertial forces. The Galilean law of inertia shows that there is a kind of obligatory guidance in the universe, which constrains a body left to itself to move with a perfectly definite motion, once it has been set in motion in a particular direction in the world. The body does this in virtue of a tendency of persistence, which carries on this direction at each instant "parallel to itself." At every position $\mathrm{P}$ in the universe, this tendency of persistence (the "guiding field") thus determines the infinitesimal parallel displacement of vectors from $P$ to world-points indefinitely near to $\mathrm{P}$. Such a continuum, in which this idea of infinitesimal parallel displacement is determinate, I have designated as an "affinely connected" one (affin zusammenhängend). According to the ideas of Galileo and Newton, the "affine connection" of the universe (the difference between straight and curved) is given by its geometrical structure. A vector at any position in the universe determines directly and without ambiguity, at every other position, and by 
itself (i.e. independently of the material content of the universe), a vector "equal" to itself. According to Einstein, however, the guiding field (Führungsfeld) is a physical reality which is dependent on the state of matter, and manifests itself only infinitesimally (as a tendency of persistence which carries over the vectors from one point to "indefinitely neighbouring" ones). The immense success of Einstein's theory is based on the fact that the effects of gravitation also belong to the guiding field, as we should expect a priori from our experience of the equality of gravitational and inertial mass. The planets follow exactly the orbit destined to them by the guiding field; there is no special "gravitational force" necessary, as in Newton's theory, to cause them to deviate from their Galilean orbit. In general, the parallel displacement is "non-integrable"; i.e. if we transfer a vector at $\mathrm{P}$ along two different paths to a point $\mathrm{P}^{\prime}$ at a finite distance from $P$, then the vectors, which were coincident at $\mathrm{P}$, arrive at $\mathrm{P}^{\prime}$ in two different end-positions after travelling these two paths.

The "affine connection" is not an original characteristic of the universe, but arises from a more deeply lying condition of things - the "metrical field." There exists an infinitesimal "light-cone" (Lichtkegel) at every position $\mathrm{P}$ in the world, which separates past and future in the immediate vicinity of the point P. In other words, this light-cone separates those world-points which can receive action from $P$ from those from which an "action" can arrive at P. This "cone of light" renders it possible to compare two lineelements at $\mathrm{P}$ with each other by measurement; all vectors of equal measure represent one and the same distance at $\mathrm{P}$. In addition to the determination of measure at a point $P$ (the "relation of action" of $\mathrm{P}$ with its surroundings), we have now the "metrical relation," which determines the congruent transference of an arbitrary distance at $P$ to all points indefinitely near to $P$.

Just as the point of view of Einstein leads back to that of Galileo and Newton when we assume the transference of vectors by parallel displacement to be integrable, so we fall back on Einstein when the transference of distances by congruent transference is integrable. But this particular assumption does not appear to me to be in the least justified (apart from the progress of the historical development). It appears to me rather as a gross inconsistency. For the "distances" the old point of view of a determination of magnitudes in terms of each other is maintained, this being independent of matter and taking place directly at a distance. This is just as much in conflict with the principle of the relativity of magnitude as the point of view of Newton and Galileo is with the principle of the relativity of motion. If, in the case in point, we proceed in earnest with the idea of the continuity of action, then "magnitudes of condition " occur in the mathematical description of the worldmetrics in just sufficient number and in such a combination as is necessary for the description of No. 2677 , vOL. IO6] the electromagnetic and of the gravitational field. We saw above that, besides inertia (the retention of the vector-direction), gravitation was also included in the guiding field, as a slight variation of this, as a whole, constant inertia. So in the present case, in addition to the force which conserves space- and time-lengths, electromagnetism is also included in the metrical relation. Unfortunately, this cannot be made clear so readily as in the case of gravitation. For the phenomena of gravitation are easily obtained from the Galilean principle, according to which the world-direction of a mass-point in motion follows at every instant the parallel displacement. Now it is by no means the case that the ponderomotive force of the electromagnetic field should be included in our Galilean law of motion, as well as gravitation, for a charged mass-point does not follow the guiding field. On the contrary, the correct equations of motion are obtained only by the establishment of a definite and concrete law of Nature, which is possible within the framework of the theory, and not from the general principles of the theory.

The form of the law of Nature on which the condition of the metrical field is dependent is limited by our conception of the nature of gravitation and electricity in still greater measure than it is by Einstein's general principle of relativity. When the metrical connection alone is virtually varied, the most simple of the assumptions possible leads exactly to the theory of Maxwell. Thus, whereas Einstein's theory of gravitation gave certain inappreciable deviations from the Newtonian theory, such as could be tested by experiment, our interpretation of electricity-one is almost tempted to say unfortunately-results in the complete confirmation of Maxwell's laws. If we supplement Maxwell's "magnitude of action" (Wirkungsgrösse) by the simplest additional term which also allows of the virtual variation of the "relation of action," we then arrive at Einstein's laws of the gravitational field, from which, however, there are two small deviations :

(I) That cosmological term appears which Einstein appended later to his equations, and which results in the spatial closure (Geschlossenheit) of the universe. A hypothesis conceived ad hoc by Einstein to explain the generally prevailing equilibrium of masses results here of necessity. Whereas Einstein has to assume a pre-stabilised harmony between the "cosmological constant" which is characteristic for his modified law of gravitation, and the total mass fortuitously present in the universe, in our case, where no such constant occurs, the world-mass determines the curvature of the universe in virtue of the laws of equilibrium. Only in this way, it appears to me, is Einstein's cosmology at all possible from a physical point of view.

(2) In the case where an electromagnetic field is present, Einstein's cosmological term must be supplemented by an additional term of similar 
character. This renders the existence of charged material particles possible without requiring an immense mass-horizon as in Einstein's cosmology.

At first the non-integrability of the transference of distances (Streckenübertragung) aroused much antipathy. Does not this mean that two measuring-rods which coincide at one position in the universe no longer need to coincide in the event of a subsequent encounter? Or that two clocks which set out from one world-position with the same period will possess different periods should they happen to encounter at a subsequent position in space? Such a behaviour of "atomic clocks " obviously stands in opposition to the fact that atoms emit spectral lines of a definite frequency, independently of their past history. Neither does a measuring-rod at rest in a static field experience a congruent transference from moment to moment.

What is the cause of this discrepancy between the idea of congruent transfer and the behaviour of measuring-rods and clocks? I differentiate between the determination of a magnitude in Nature by "persistence" (Beharrung) and by "adjustment" (Einstellung). I shall make the difference clear by the following illustration: $\mathrm{We}$ can give to the axis of a rotating top any arbitrary direction in space. This arbitrary original direction then determines for all time the direction of the axis of the top when left to itself, by means of a tendency of persistence which operates from moment to moment; the axis experiences at every instant a parallel displacement. The exact opposite is the case for a magnetic needle in a magnetic field. Its direction is determined at each instant independently of the condition of the system at other instants by the fact that, in virtue of its constitution, the system adjusts itself in an unequivocally determined manner to the field in which it is situated. $\Lambda$ priori we have no ground for assuming as integrable a transfer which results purely from the tendency of persistence. Even if that is the case, as, for instance, for the rotation of the top in Euclidean space, we should find that two tops which start out from the same point with the same axial positions and encounter again after the lapse of a very long time would show arbitrary deviations of their axial positions, for they can never be completely isolated from every influence. Thus, although, for example, Maxwell's equations demand the conservational equation $d e / d t=0$ for the charge $e$ of an electron, we are unable to understand from this fact why an electron, even after an indefinitely long time, always possesses an unaltered charge, and why the same charge $e$ is associated with all electrons. This circumstance shows that the charge is not determined by persistence, but by adjustment, and that there can exist only one state of equilibrium of the negative electricity, to which the corpuscle adjusts itself afresh at every instant. For the same reason we can conclude the same thing for the spectral lines of atoms. The one thing common to atoms emitting the same frequency is their constitution, and not the agreement of their frequencies on the occasion of an encounter in the distant past. Similarly, the length of a measuring-rod is obviously determined by adjustment, for I could not give this measuring-rod in this field-position any other length arbitrarily (say double or treble length) in place of the length which it now possesses, in the manner in which I can at will predetermine its direction. The theoretical possibility of a determination of length by adjustment is given as a consequence of the world-curvature, which arises from the metrical field according to a complicated mathematical law. As a result of its constitution, the measuring-rod assumes a length which possesses this or that value, in relation to the radius of curvature of the field. In point of fact, and taking the laws of Nature indicated above as a basis, it can be made plausible that measuring-rods and clocks adjust themselves exactly in this way, although this assumptionwhich, in the neighbourhood of large masses, involves the displacement of spectral lines towards the red upheld by Einstein-does not appear anything like so conclusive in our theory as it does in that of Einstein.

\section{The Relativity of Time.}

By Prof. A. S. Eddington, F.R.S.

THE philosopher discusses the significance of 1 time; the astronomer measures time. The astronomer goes confidently about his business and does not think of asking the philosopher what exactly is this thing he is supposed to be measuring; nor does the philosopher always stop to consider whether time in his speculations is identical with the time which the world humbly accepts from the astronomer. In these circumstances it is not surprising that some confusion should have arisen.

In many globular clusters there are stars which oscillate in intrinsic brightness; let us select two such stars from different clusters and invite all NO. 2677 , VOL. IO6] the astronomers in the universe to measure the true interval of time between the moments of maximum light of the two stars. They must, of course, make whatever measurements and calculations they consider necessary to allow for the finite velocity of light. It may easily happen that the astronomers on Arcturus report that the two maxima were simultaneous; whereas those on the earth report an interval of ten years between the same two maxima. There is here no question of observational error; the recognised terrestrial method necessarily gives a discordant result when used on Arcturus, owing to its different motion.

Our first impulse is to blame the astronomers. 